\title{
Structure of the WD40-domain of human ATG16L1
}

\author{
Milica Bajagic, Archna Archna, Petra Büsing, and Andrea Scrima* \\ Structural Biology of Autophagy Group, Department of Structure and Function of Proteins, Helmholtz Centre for Infection \\ Research, Braunschweig, 38124, Germany
}

Received 23 May 2017; Accepted 29 June 2017

DOI: $10.1002 /$ pro.3222

Published online 7 July 2017 proteinscience.org

\begin{abstract}
Autophagy-related protein ATG16L1 is a component of the mammalian ATG12 ATG5/ ATG16L1 complex, which acts as E3-ligase to catalyze lipidation of LC3 during autophagosome biogenesis. The N-terminal part of ATG16L1 comprises the ATG5-binding site and coiled-coil dimerization domain, both also present in yeast ATG16 and essential for bulk and starvation induced autophagy. While absent in yeast ATG16, mammalian ATG16L1 further contains a predicted C-terminal WD40-domain, which has been shown to be involved in mediating interaction with diverse factors in the context of alternative functions of autophagy, such as inflammatory control and xenophagy. In this work, we provide detailed information on the domain boundaries of the WD40-domain of human ATG16L1 and present its crystal structure at a resolution of $1.55 \AA$.
\end{abstract}

Keywords: ATG16L1; WD40; seven-bladed $\beta$-propeller; X-ray crystallography; protein-protein interaction; autophagy

\section{Introduction}

Macroautophagy (hereafter referred to as autophagy) is a conserved eukaryotic mechanism for the sequestration and degradation of cytoplasmic constituents. Activation by nutrient depletion or stress

Abbreviations: ATG, autophagy-related genes; $\mathrm{BD}$, binding domain; CCD, coiled-coil domain; DDB1, DNA-damagebinding protein 1; EED, embryonic ectoderm development; Ins., insert region; PE, phosphatidylethanolamine; SER, surface entropy reduction; WDD, WD40 propeller domain

Additional Supporting Information may be found in the online version of this article.

Statement for Broader Audience Autophagy is a conserved cellular mechanism for the degradation and recycling of intracellular components and plays an important role in immune defense by detecting and eliminating intracellular pathogens. Defective autophagy can give rise to severe diseases such as cancer, neurodegeneration and Crohn's Disease. One of the key regulators of autophagy is ATG16L1. In this work we describe the first structure of the WD40-domain of ATG16L1, which will help to improve our understanding of autophagy regulation.

${ }^{*}$ Correspondence to: Andrea Scrima, Structural Biology of Autophagy Group, Department of Structure and Function of Proteins, Helmholtz Centre for Infection Research, 38124 Braunschweig, Germany. E-mail: andrea.scrima@helmholtz-hzi.de induces the formation of a double-membrane vesicle referred to as autophagosome, which engulfs a random section of cytoplasm and subsequently fuses with lysosomes for degradation of the sequestered material. ${ }^{1,2}$ But apart from its classical role in bulkdegradation, autophagy also mediates degradation of specific components in the cytoplasm in a process called selective autophagy. These specific targets for autophagy-mediated degradation include, among others, defective organelles, large protein aggregates and intracellular pathogens. ${ }^{3-7}$ Additionally, autophagy is involved in a large number of immunity-related processes in mammals, such as thymic selection, antigen presentation, lymphocyte homeostasis and regulation of inflammatory responses. ${ }^{5,8,9}$ The importance of autophagy is further reflected in the finding that defects in autophagy are linked to the onset of cancer, neurodegenerative and chronic inflammatory diseases. ${ }^{10-12}$ The latter include Crohn's disease, a complex chronic inflammatory disease of the intestine that causes lesions in the intestinal tract with an increased risk of colon cancer formation. ${ }^{13,14}$

Initiated by the genetic screening of macroautophagy-defective mutants in yeast, ${ }^{15,16}$ to date more than thirty autophagy-related genes (ATG) 
A

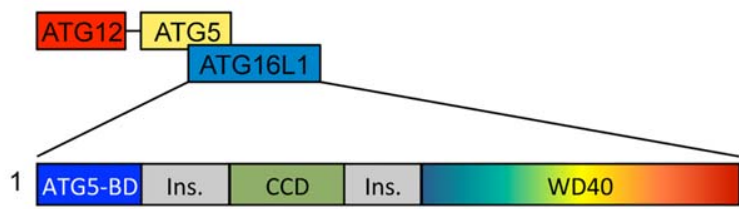

H. sapiens ATG16L1

1 ATG5-BD $\mid$ CCD 150

S. cerevisiae ATG16

B

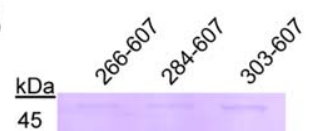

45
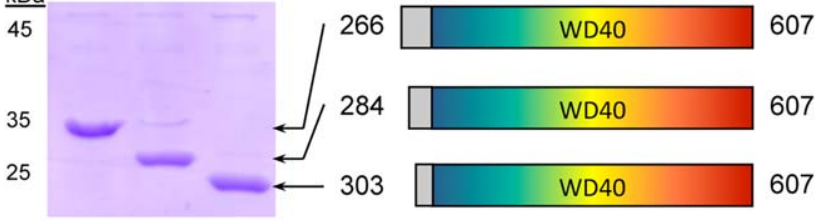
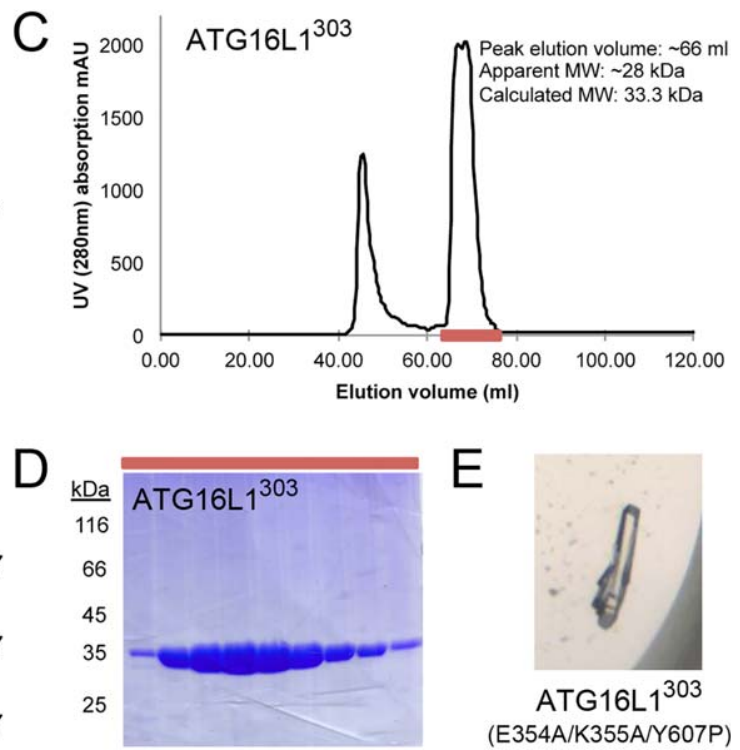

Figure 1. Domain architecture of human ATG16L1 and expression/purification/crystallization of the WDD. (A) Scheme of the domain organization of human ATG16L1 in comparison to yeast ATG16. (Ins.=insert region). (B) SDS-PAGE analysis of the three soluble WDD constructs. (C) Exemplary elution profile for ATG16L $1^{303}$ of the size exclusion chromatography run on HiLoad 16/60 Superdex 75 column. Absorbance of the protein is measured at $280 \mathrm{~nm}$. (D) Coomassie stained gel of eluted fractions analyzed by SDS-PAGE. Red bar represents the corresponding elution fractions in the chromatogram (C). (E) Crystal of ATG16L1 ${ }^{303}$ with two SER-mutations (E354A/K355A) and one human/mouse chimeric mutation (Y607P)

and their respective proteins have been identified. ATG16L1, the mammalian homolog of yeast ATG16, is a member of the core autophagy machinery and component of the E3-ligase ATG12 ATG5/ATG16L1 complex (ATG12 ATG5/ATG16 in yeast). ${ }^{17-20}$ The E3-ligase complex conjugates phosphatidylethanolamine $(\mathrm{PE})$ to LC3 (the mammalian homolog of yeast ATG8) and determines the site of conjugation during autophagosome biogenesis. ${ }^{21,22}$ Further studies on the function of the yeast E3-ligase complex showed that ATG12 ATG5/ATG16, in addition to directly binding to membranes, also binds to conjugated ATG8-PE, thereby crosslinking ATG8-PE/ATG12-ATG5 and driving the ordered assembly of a membrane scaffold that is critical for yeast autophagy. ${ }^{23,24}$

Human ATG16L1 is a 607 amino acid protein ( $\sim 68 \mathrm{kDa}$ ) comprising three major domains: the N-terminal ATG5 binding domain (ATG5-BD), the central coiled-coil domain (CCD) and a predicted C-terminal WD40-domain [Fig. 1(A)]. A short helix on the N-terminal part of the mammalian ATG16L1, also present in the 150 amino acid yeast homolog ATG16, forms the ATG5-BD, and its structure has previously been determined in complex with the human ATG12 ATG5 conjugate. ${ }^{25}$ Apart from the ATG5-BD, mammalian ATG16L1 and yeast ATG16, both comprise a central coiled-coil domain (CCD), important for oligomerization of the protein. The crystal structure of the yeast ATG16-CCD reveals a parallel dimeric arrangement of the coiled-coil-helices, suggesting a parallel orientation of full-length ATG16/ ATG16L1 molecules in the dimer, ${ }^{26}$ which is further supported by the detection of a dimeric state of human ATG16L1-CCD in solution. ${ }^{27}$ In addition to being important for homo-dimerization of ATG16L1, the CCD in human ATG16L1 also acts as interaction site for various proteins involved in the autophagy process, such as WIPI2 and Rab33, a small Golgi-resident GTPase. ${ }^{28,29}$ Both WIPI2 and Rab33 serve as modulators of autophagosome elongation through interaction with ATG16L1. Additionally, FIP200, a member of the ULK autophagy initiation complex, binds ATG16L1 in the region between the CCD and the predicted WD40domain. ${ }^{30}$ While ATG5BD and CCD are common to both yeast ATG16 and human ATG16L1, the major difference to the yeast protein is a predicted WD40 propeller domain (WDD) on the C-terminal part, which is exclusively present in mammalian ATG16L1. The absence of this domain from the yeast protein indicates that it is not essential for bulk autophagy, in line with the observation that a truncated mouse ATG16L1-variant lacking the WDD reconstitutes canonical autophagy in ATG16L1-deficient cells. ${ }^{31}$ Notably, the stretch between CCD and predicted WD40-domain of ATG16L1 also harbors the position of a natural variant of ATG16L1 (T300A), a risk factor for Crohn's disease ${ }^{32}$ and cleavage site by caspase 3 and caspase $7,,^{33,34}$ that results in a decreased level of selective antibacterial autophagy and elevated levels of inflammatory response. When cleavage occurs at position 300, the C-terminal WDD domain is separated from the full-length protein and despite the presence of both fragments, canonical autophagy is not affected, whereas unconventional, selective 
autophagy is largely inhibited. ${ }^{35}$ The WDD is thus not required for canonical bulk autophagy, but activation of unconventional, selective autophagy appears to require an intact ATG16L1 protein. The family of WD40 propeller domains is very common in eukaryotic systems and often provides a protein-protein/ ligand interaction platform to regulate different cellular processes. The mammalian ATG16L1 might thus have acquired the WDD during evolution to provide a protein-protein interaction platform for the regulation of specific needs of higher eukaryotic systems, such as inflammatory control and clearance of pathogens. To further support this line of reasoning, proteins mentioned so far in the concept of linking autophagy with pathogen clearance, including the NOD-like receptor protein NOD2, Toll-like receptor TLR2 and the transmembrane protein TMEM59, have been shown to bind to the WDD of ATG16L1 via a short ATG16L1binding motif. ${ }^{36}$ In the context of pathogen clearance, NOD-like receptors NOD1 and NOD2 have been shown direct autophagy to the site of bacterial entry through interaction with ATG16L1. ${ }^{37,38}$ Apart from these, the WDD of ATG16L1 has been shown to further interact with Ubiquitin, ${ }^{39}$ the DNA-damagebinding protein DDB1, ${ }^{40}$ which is part of a large Cullin-E3 ubiquitin ligase complex, the transmembrane protein TMEM166/EVA1A, ${ }^{41}$ TRIM20, a member of the multifunctional tripartite motif family of proteins, ${ }^{42}$ as well as T3JAM and DEDD2, two proteins haroboring the previously identified ATG16L1motif. ${ }^{36}$ Given the ever growing importance selective autophagy has in the mammalian system, ATG16L1, and more specifically the predicted WDD of ATG16L1, provides a direct link between autophagy and other cellular pathways. Despite its important function in acting as a mammalian-specific protein-interaction platform, so far no structural information is available for the predicted C-terminal WDD of ATG16L1.

\section{Results}

\section{Identification of soluble WDD-constructs}

In the work presented here, we thus aimed at obtaining structural information for the predicted Cterminal WDD of human ATG16L1. To determine the crystal structures of the WDD, we initially analyzed the sequence of human ATG16L1 (UniprotQ676U5) using PROSITE, ${ }^{43}$ PFAM $^{44}{ }^{4 M A R T}{ }^{45}$ and PSIPRED $^{46}$ to identify the boundaries of the WDD. Based on these predictions, the C-terminal WDD encompasses residues 318-607 (PROSITE), 313-604 (PFAM) or 311-505 (SMART) comprising five to seven predicted WD40-blades. PSIPRED further predicts the presence of $\beta$-strands at position 286-290 and 308-309. Notably, residues 266-284 are missing in splice isoform 2 of ATG16L1, identifying residues 266 and 284 as further potential domain boundaries for soluble WDD expression constructs. Based on this sequence analysis, we chose three $\mathrm{N}$-terminal boundaries for the recombinant expression of soluble WDD: residue G303, which is located in a predicted flexible GSG-motif, has been chosen as N-terminal boundary for the expression of soluble WDD (human ATG16L1 303-607 will hereafter be referred to as ATG16L1 $1^{303}$ ), and two additional expression constructs with $\mathrm{N}$-terminal boundaries at residues K266 and G284 have been chosen as additional constructs extending $\mathrm{N}$-terminally beyond the predicted WDD [Fig. 1(B)]. Since initial expression of these constructs in $E$. coli resulted in insoluble protein, we utilized the Baculovirus insect cell expression system, yielding approx. $1-3 \mathrm{mg} / 4 \mathrm{~L}$ culture of soluble and pure protein. All three constructs were monomeric in solution, as estimated by the apparent molecular weight of the protein derived for the elution volume on size-exclusion chromatography [Fig. 1(C,D)]. Additionally, we used a construct comprising the commonly used WDD boundaries with residues $320-607$, for which we could however not detect any soluble protein (not shown).

\section{Crystallization and structure determination}

All three soluble constructs were used for crystallization trials, but did not yield crystals. As crystallization is highly dependent on the surface of the target protein, we used surface entropy reduction (SER) mutants to improve crystallization behavior. ${ }^{47}$ For all three constructs, we generated four different surface entropy reduction mutants (K306A/E307A, E354A/K355A, K469A/K470A, E569A/K570A), which however failed to crystallize as well. Mouse (Mus Musculus) ATG16L1 (UNIPROT-Q8C0J2) shares 94\% sequence identity (96\% protein sequence similarity) with human ATG16L1, suggesting that variable residues are likely to be located on the protein surface and not to influence stability or folding. Since the SER-mutants failed to crystallize, we combined these with single point mutants to generate human-mouse chimeric versions of ATG16L1. Of these, the human ATG16L1 ${ }^{303}$ with the triple mutation E354A/K355A/Y607P resulted in crystals diffracting to $1.55 \AA$ resolution [Fig. 1(E), Supporting Information Figs. S1 and S2, Table I).

\section{Structure of the WDD of human ATG16L1}

The high-resolution structure of the C-terminal domain of ATG16L1 reveals a seven-bladed WD40propeller domain [Fig. 2(A)]. Each blade comprises the canonical anti-parallel $\beta$-sheet composed of four $\beta$-strands A-D, with the exception of blade 6 , where residues at the canonical position of strand $A$ do not adopt a $\beta$-strand conformation. The strands D-A-B-C harbor the WD40 repeat sequence, and the WD40 repeat is thus not equivalent to one blade. In the WDD of ATG16L1, residues of the most N-terminal strand (313-319) form strand D of blade 7, which is 
Table I. Data Collection and Refinement Statistics

\begin{tabular}{|c|c|}
\hline Data collection & $\begin{array}{l}\text { ATG16L1-WDD } \\
\text { (PDB: 5NUV) }\end{array}$ \\
\hline X-ray source & BL 14.2, BESSY \\
\hline Wavelength $[\AA]$ & 0.9184 \\
\hline Resolution $[\AA ̊ \bar{c}]$ & $43.9-1.55(1.65-1.55)$ \\
\hline Space group & $\mathrm{C} 2$ \\
\hline \multicolumn{2}{|l|}{ Cell dimensions } \\
\hline $\mathrm{a}, \mathrm{b}, \mathrm{c}[\AA]$ & $157.55,38.22,45.62$ \\
\hline$\alpha, \beta, \gamma\left[{ }^{\circ}\right]$ & $90.00,105.7490 .00$ \\
\hline$R_{\text {merge }}[\%]$ & $6.7(73.0)$ \\
\hline $\mathrm{R}_{\text {meas }}[\%]$ & $7.3(79.2)$ \\
\hline $\mathrm{CC} 1 / 2[\%]$ & $99.9(82.7)$ \\
\hline$\langle\mathrm{I} / \sigma(\mathrm{I})\rangle$ & $15.6(2.4)$ \\
\hline Completeness [\%] & $99.8(99.6)$ \\
\hline Unique reflections & $38280(6488)$ \\
\hline Redundancy & $6.7(6.7)$ \\
\hline \multicolumn{2}{|l|}{ REFINEMENT } \\
\hline Resolution $[\AA]$ & $43.9-1.55(1.59-1.55)$ \\
\hline No. of reflections & 38278 \\
\hline$R_{\text {work }}$ & $0.165(0.278)$ \\
\hline$R_{\text {free }}$ & $0.197(0.324)$ \\
\hline $\begin{array}{l}\text { No. of atoms (protein/ } \\
\text { ligand/solvent) }\end{array}$ & $2316 / 16 / 220$ \\
\hline Avg. $B$ factor protein $\left[\AA^{2}\right]$ & 26.7 \\
\hline \multicolumn{2}{|l|}{ R.m.s. deviations } \\
\hline Bond length [̊̊] & 0.009 \\
\hline Bond angles $\left[{ }^{\circ}\right]$ & 1.108 \\
\hline $\begin{array}{l}\text { Ramachandran [\%] } \\
\text { (favored/allowed/disallowed) }\end{array}$ & $96.72 / 2.95 / 0.33$ \\
\hline
\end{tabular}

Values in parentheses refer to the highest resolution shell.

$R_{\text {merge }}=\frac{\sum_{h k l} \sum_{j}\left|I_{h l l, j}-\left\langle I_{h l l}\right\rangle\right|}{\sum_{h k l} \sum_{j} I_{h l l}, j}$ and $\quad R_{\text {meas }}=\frac{\sum_{h k l} \sqrt{\frac{n}{n-1}} \sum_{j=1}^{n}\left|I_{h k l, j}-\left\langle I_{h l l}\right\rangle\right|}{\sum_{h k l} \sum_{j} I_{h k l, j}}$. With $I$ being the measured intensity and being the averaged intensity of each unique reflection with indices $h k l . I / \sigma(I)$ corresponds to the average of the intensity divided by its average standard deviation. $R_{\text {work } / \text { free }}=\frac{\sum_{h k l}\left|F_{h k l}^{\text {obs }}-F_{h k l}^{\text {calc }}\right|}{\sum_{h k l} F_{h k l}^{\text {bh }}}$ where $F_{\text {obs }}$ and $F_{\text {calc }}$ are the observed and calculated structure factors, respectively. $R_{\text {free }}$ is the same as $R_{\text {work }}$, calculated for the $5 \%$ of the data that was randomly omitted from refinement.

completed by the interaction with the very $\mathrm{C}$ terminal strands A-C of blade 7 [Fig. 2(A,B)]. Thereby, the N-terminal strand $7 \mathrm{D}$ also acts as a latch that closes the ring-shaped propeller structure. In line with that function, the observation that construct 320-607 yields no soluble protein indicates that lack of strand 7D destabilizes the fold and results in reduced solubility and degradation.

\section{Discussion}

Regarding the specific function of the ATG16L1 WDD, proteins containing a WD40-domain are widely present in eukaryotes and are involved in various cellular processes including gene regulation, development, signal transduction, vesicular transport, cell cycle control, apoptosis and chromatin dynamics. $^{48,49}$ But despite of the conserved fold, WD40-domains only share low sequence conservation, hampering the identification and assignment of the individual repeats, ${ }^{50}$ as also seen for the WDD of human ATG16L1. Individual proteins with enzymatically active WD40-domains have been reported, ${ }^{51}$ but the main function of all WD40domains described so far is to mediate interactions with other proteins, peptides or nucleic acids. The typical seven-bladed WD40-domain utilizes three surfaces for such interactions, the top/narrow, the bottow/wide surface and the whole circumference of the ring-shaped propeller [Figs. 2(A) and 3(A)]. These three surfaces are formed by two main variable regions in each blade, which are not essential for the overall stability of the fold, but provide interaction specificity and function to the WD40-domain. The largest variable region ' $\mathrm{I}$ ' is located between strand $\mathrm{C}$ of one blade and strand $\mathrm{A}$ of the next (including strand D) and typically includes $\sim 20$ residues, but can vary between 11 and 150 residues in length. While strand $\mathrm{D}$ of variable region ' $\mathrm{I}$ ' is exposed to the outside surface of the propeller and forms the circumference, the loop between strands D and A of variable region 'I' together with the loop connecting $\mathrm{B}$ and $\mathrm{C}$, form the top/narrow surface. The second variable region 'II' comprises the loop between strand $\mathrm{A}$ and $\mathrm{B}$ and is generally 2-30 residues in length and exposed at the bottom/wide end of the propeller. ${ }^{52}$ Thereby, the WD40-domain, despite representing a conserved fold with high stability, at the same time allows for high versatility derived from the variable regions and the respective large variable surface they form. For some proteins, these variable regions are sufficiently long to include independently folded domains, which however is not the case for the ATG16L1 WDD.

To identify evolutionarily important regions within the WDD that might be involved in mediating interactions, the amino acid conservation has been analyzed by NCBI Blast using the sequence of the human ATG16L1-WDD (307-607). Of these NCBI Blast hits, the top 90 sequences from different organisms share identities above $92 \%$. These have been used for an analysis using Consurf [Fig. 3(B), Supporting Information 2], ${ }^{53}$ that estimates the evolutionary conservation based on the phylogenetic relations between homologous sequences. The Consurf analysis of the top 90 NCBI Blast hits shows an evolutionary conservation of the variable regions I and II forming the top, side and bottom of blades 23 [Fig. 3(B)]. In contrast to the high degree of conservation in blades 2 and 3, conservation in blades 4 and 5 is mainly restricted to the top and bottom surface with variability on the side. Apart from conserved residues in the WDD core (involved in stabilizing the WD40-fold) the outer top, bottom and side surfaces of blades 1, 6 and 7 show a higher variability as compared to the rest of the WDD. As seen for various WD40-domains, a tunnel is present in the center of the propeller-shaped WDD, which in 

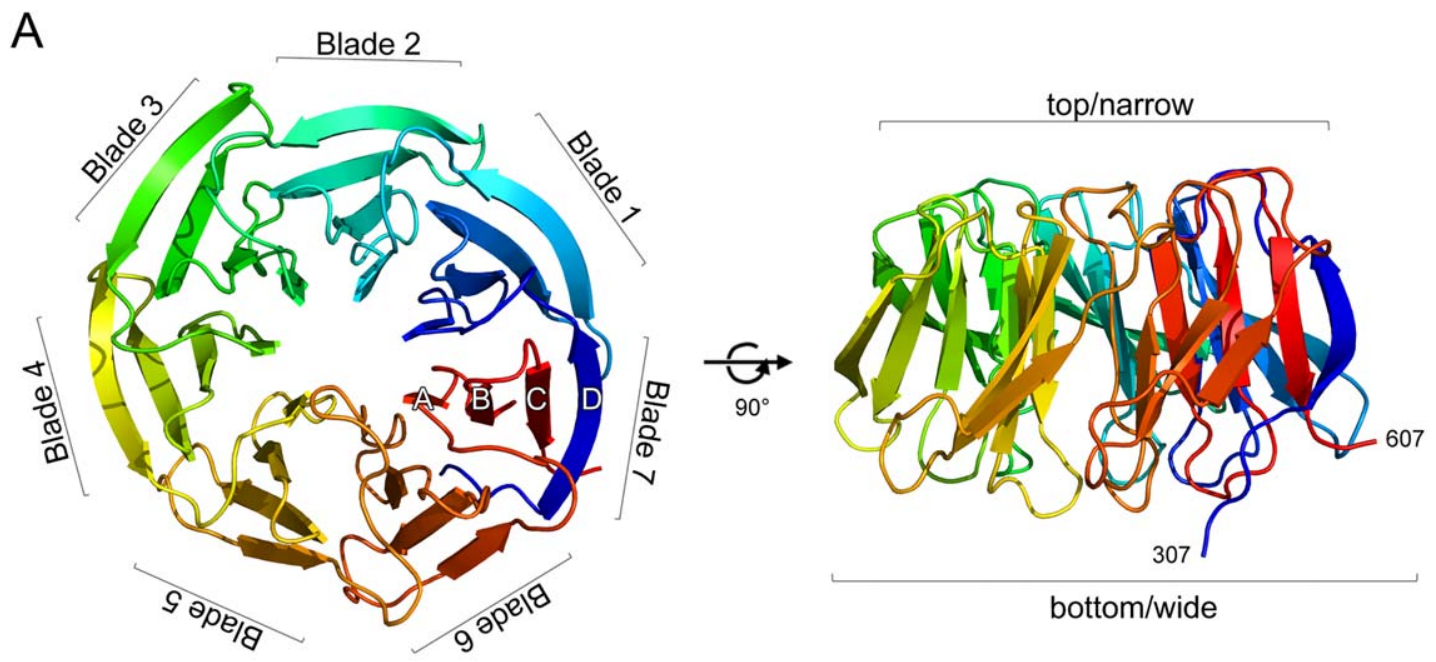

B

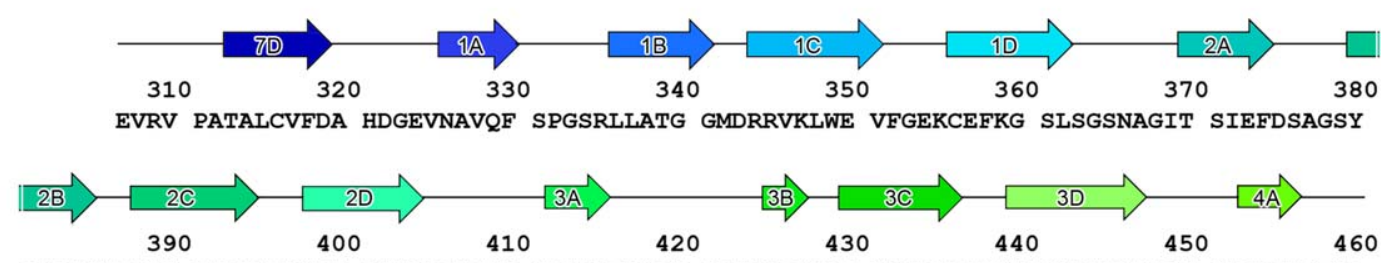
LLAASNDFAS RIWTVDDYRL RHTLTGHSGK VLSAKFLLDN ARIVSGSHDR TLKLWDLRSK VCIKTVFAGS SCNDIVCTEQ

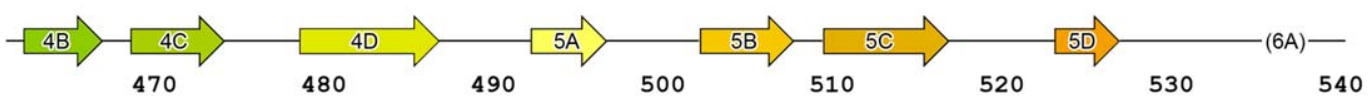
CVMSGHFDKK IRFWDIRSES IVREMELLGK ITALDLNPER TELLSCSRDD LLKVIDLRTN AIKQTFSAPG FKCGSDWTRV

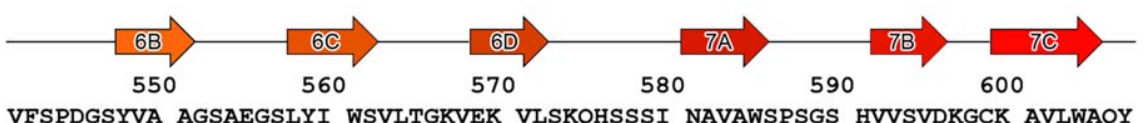

Figure 2. Structure of the ATG16L1 WDD. (A) Cartoon representation of the structure of ATG16L1 WDD in two orientations. The seven-bladed propeller is shown in rainbow color ( $N$ terminus in blue, $C$ terminus in red). Individual strands (A-D) and blades (1-7) are labeled. (B) Sequence of the ATG16L1 WDD (corresponding to the wild-type human ATG16L1 sequence) with the assignment of secondary structure boundaries

ATG16L1 WDD is non-continuous and separated by the Arg539 side chain into a shallow, top tunnel of approx. $8 \AA$ in length and an approx. $19 \AA$ deep bottom tunnel [Fig. 3(B-F)]. Apart from the conservation in the outer surface, evolutionarily conserved regions also comprise residues in strand A (or its equivalent region) of all seven blades forming the inner cavity of the top and bottom tunnel, as well the opening of the tunnel on the WDD top and bottom surface.

Apart from the surface conservation, we analyzed the charge distribution on the WDD surface. In ATG16L1 WDD, the top and bottom surface show a distinct charge distribution, with a basic top and an acidic bottom surface [Fig. 3(C)]. The top tunnel is mainly hydrophobic [Fig. 3(D,E)], whereas the bottom tunnel is negatively charged [Fig. 3(E,F)]. Notably, an additional positively charged cavity is present between blades 3 and 4, which however is only accessible via two narrow channels from the bottom and the side [Fig. 3(E), Supporting Information Fig. S4].
Based on the analysis of evolutionarily conserved regions performed with Consurf, functional regions that are likely to be involved in mediating interactions via the WDD cluster on the top and bottom tunnel as well as on the whole exposed surface of blades 2-3 and the top and bottom surfaces of blades $4-5$. Furthermore, the distinct charge distribution on the top and bottom interaction surfaces (including the tunnel and the positively charged cavity) might be required for the recognition of putative subsets of known ATG16L1-WDD-binding proteins, such as the specific recognition of the previously reported ATG16L1-binding motif, ${ }^{36}$ or yet to be identified interaction partners. In that regard, the high conservation in the top and bottom tunnel is reminiscent of WD40-domains that utilize the tunnel for specific recognition, such as in the case of EED, which is involved in the readout of Histone3 lysine methylation patterns and binds an arginine residue in the central tunnel. ${ }^{54}$ It is thus not unlikely, that ATG16L1-WDD might utilize the top/bottom tunnel 

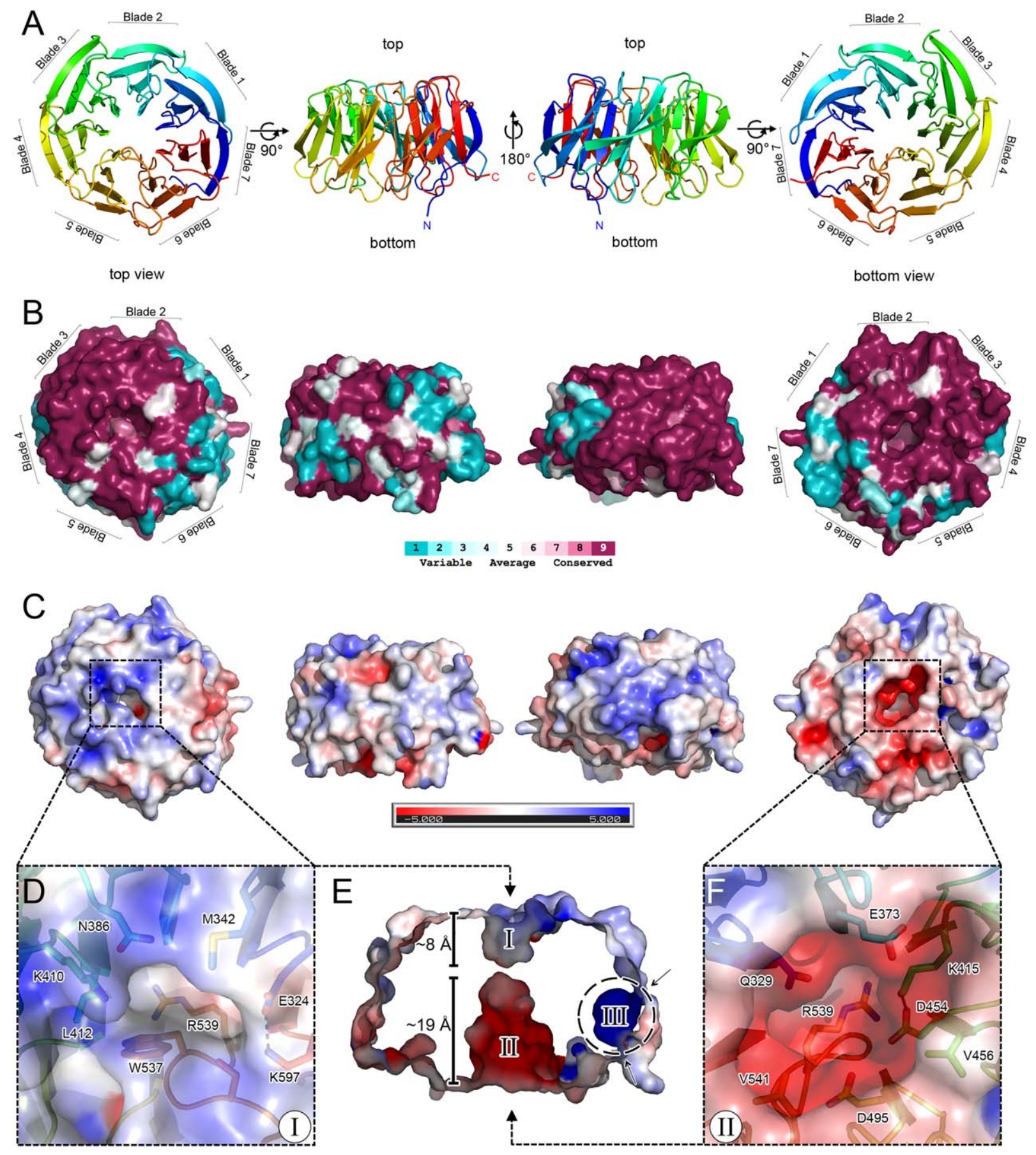

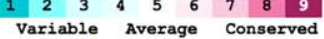

Figure 3. Conservation and electrostatic surface properties of the WDD. (A) Cartoon representation of the structure of ATG16L1 WDD in four orientations. (B) Evolutionary conservation of residues in the WDD analyzed by Consurf and plotted onto the WDD surface. 90 sequences obtained from a NCBI Blast search with the ATG16L1-WDD sequence (307-607) were used for the analysis (see also Supporting Information Fig. S3 and Supporting Information 2). (C) Electrostatic surface potential of the WDD shown in the same orientation as in (A) (from $-5 \mathrm{kT} / \mathrm{e}$ (red) to $+5 \mathrm{kT} / \mathrm{e}$ (blue), model was changed to wild-type ATG16L1 sequence for the purpose of electrostatic potential calculation). (D) View onto the shallow, top tunnel (I). (E) Longitudinal section of the electrostatic surface, depicting the dimensions and location of the top tunnel (I), bottom tunnel (II) and basic cavity (III). Dashed arrows represent the respective view onto the top (I) and bottom (II) tunnel as shown in (D) and (F). Solid arrows indicate the points at which the basic cavity (III) is accessible through narrow solvent channels (see also Supporting Information Fig. S4). (F) View onto the deep, bottom tunnel (II)

for specific recognition of posttranslational modifications, that could control the interaction between the WDD and interaction partners. Overall, the evolutionarily conserved region covers a large area of the
WDD surface, extending from the bottom, over the side to the top of the WDD. Rather than representing a single interaction surface, this suggests that the WDD harbors several separate conserved 

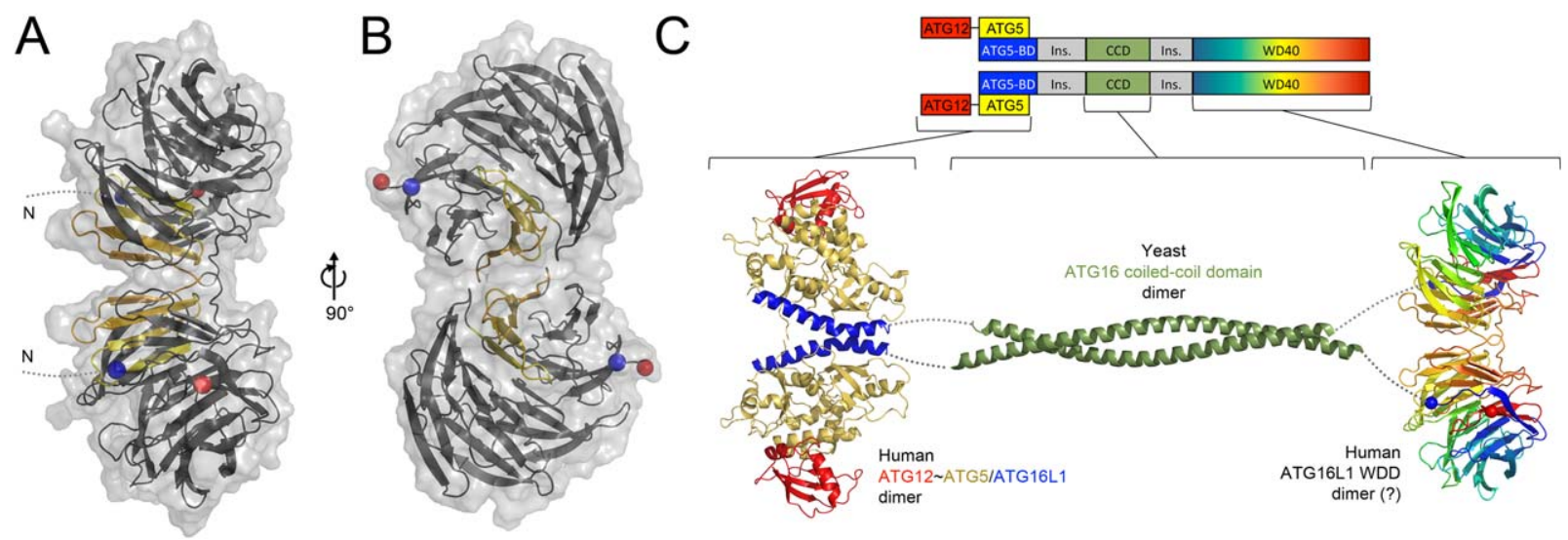

Figure 4. Putative dimer of the WDD. (A) and (B) Cartoon and surface representation of dimeric WDD observed in the crystal in two orientations. Dimerization mainly involves blade 5 (in yellow), with additional contributions from blades 4 and 6 . The total interface area comprises $620 \AA^{2}\left(\Delta^{\mathrm{i}} \mathrm{G}=-7.6 \mathrm{kcal} / \mathrm{mol}, \Delta^{\mathrm{i}} \mathrm{G} P\right.$-value $\left.=0.112\right) . \mathrm{N}$ and $\mathrm{C}$ termini are shown as blue and red sphere, respectively. The $\mathrm{N}$ termini of both WDD in the dimer extend in the same direction (dotted line). (C) Top: Schematic representation of dimerized human ATG16L1 in complex with ATG12 ATG5. Bottom: Cartoon representation of the dimeric structures of human ATG12 ATG5/ATG16L1 (PDB: 4GDK), ${ }^{25}$ yeast ATG16 CCD (PDB: 3A7P) ${ }^{26}$ and the putative WDD dimer of human ATG16L1. The orientation of the WDD dimer corresponds to the orientation seen in panel (A)

interaction surfaces, in line with the diverse interaction partners of WDD reported so far. However, while the WDD structure provides important information on the conserved regions and surface charge distribution, a detailed analysis of specific interactions with subsets of the reported WDD-interaction partners requires further structural studies of complexes and WDD-structure driven mutagenesis of conserved surface residues.

Interestingly, taking crystal-symmetry and packing into account, the WDD in the crystal forms a dimer with a neighboring symmetry related molecule. Both molecules in the dimer are arranged in a parallel fashion, with both $\mathrm{N}$ termini extending in the same direction [Fig. 4(A,B)]. The dimer interface comprises a total interface area of $620 \AA^{2}$ and mainly involves residues in blade 5 (R508, D509, D510, L511, K513, I522, Q524, F526, S527) and in loops connecting blade 5 with the neighboring blades 4 (E486, L487, L488, G489, K490) and 6 (A528, P529, K532). 13 out of the 17 residues involved in the dimerization show a high evolutionary conservation (Supporting Information Fig. S3), which might be indicative of a physiological role for dimerization of the WDD. The previously determined structures of the human ATG12 ATG5/ATG16L1 complex and the CCD of yeast ATG16 both reveal a parallel dimeric arrangement mediated via the ATG5BD and the CCD of ATG16L1/ATG16. ${ }^{25,26}$ Even though the isolated WDD appears to be monomeric in solution [Fig. 1(C)], in line with the previous observations of a dimeric ATG16L1, the dimerization of ATG5BD and especially of the CCD might potentially facilitate or induce a physiologically relevant dimerization of WDD in full-length ATG16L1 as seen in the crystal [Fig. $4(\mathrm{C})$ ].
In regard to the $\mathrm{T} 300 \mathrm{~A}$ mutation, we initially attempted to obtain further information on the so far structurally uncharacterized region containing the Crohn's disease risk polymorphism by extending the expression constructs $\mathrm{N}$-terminally beyond the predicted WDD (Fig. 1). But even though crystallization attempts using longer constructs failed and the T300 region is not covered by the WDD structure presented here, the structure still provides the important information that, in contrast to previous predictions, ${ }^{32}$ the T300A position is not part of the WDD and thus is unlikely to have an impact on the structure of the WDD. Instead, T300A is located in the region linking the coiled-coil domain and the WDD, seven amino acids N-terminal to the first amino acid defined in the WDD crystal structure. As reported before, T300A increases ATG16L1 sensitization to caspase-3/7-mediated processing. ${ }^{33,34}$ The amino acid sequence ${ }^{296} \mathrm{DNVD}^{299}$ flanks the T300 position and resembles the consensus motif DxxD for caspases 3 and 7. This region in ATG16L1 is predicted to lack any secondary structure (Supporting Information Fig. S5) and is therefore suitable for caspase-mediated cleavage, as caspases prefer an extended and unstructured substrate peptide conformation. Therefore, T300A converts the poor substrate sequence ${ }^{296} \mathrm{DxxDT}^{300}$ into ${ }^{296} \mathrm{DxxDA}^{300}$, corresponding to the caspase $3 / 7$ consensus sequence $\operatorname{DxxD}(\mathrm{G} / \mathrm{S} / \mathrm{A}){ }^{55}$ Based on the current structural knowledge, the T300A mutation is unlikely to influence the WDD structure or even the overall domain structure of full-length ATG16L1. However, recent studies uncovered that the T300A mutation impairs interaction of the WDD with a subset of interaction partners in the absence of caspase-mediated processing. ${ }^{35}$ This finding gives rise to the notion, that 


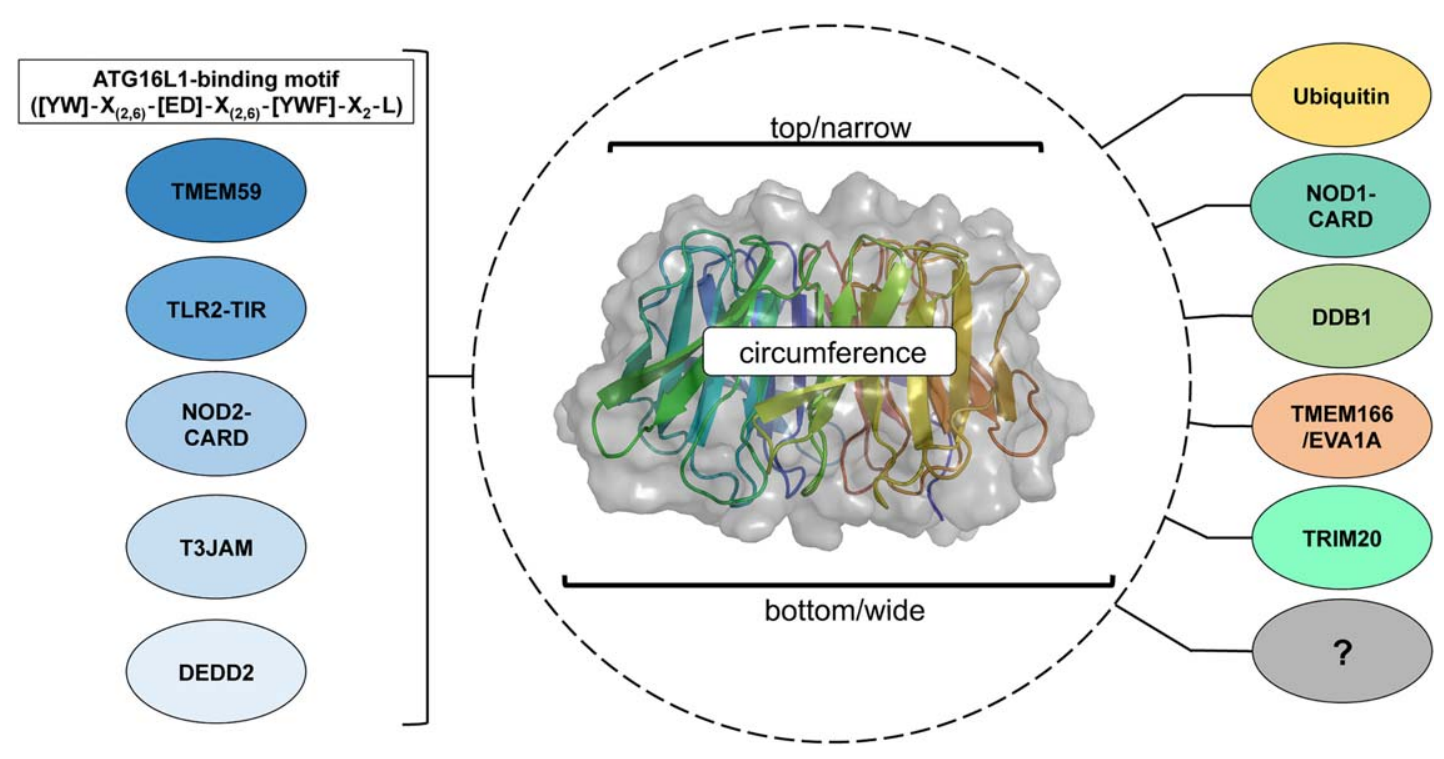

Figure 5. Schematic representation of the putative interaction surfaces on ATG16L1 WDD and its known interaction partners. The ATG16L1 WDD provides three putative interaction surface, the top/narrow and bottom/wide surface, as well as the whole circumference of the ring-shaped propeller structure. To which of the three surfaces the known and yet unidentified interaction partners bind to remains still elusive

apart from sensitizing ATG16L1 to caspase-3/7-mediated processing, T300 might also be involved in stabilizing an intramolecular (or in the ATG16L1-dimer an intermolecular) interaction of the ${ }^{296} \mathrm{DNVD}^{299}$ region with the WDD. On the molecular level, mutation of T300 to alanine may disrupt that interaction, leading to altered interaction properties of the WDD and in parallel exposing the ${ }^{296} \mathrm{DNVD}^{299}$ that would result in an increased caspase-3/7-mediated processing. Due to the lack of structural data for the ${ }^{296} \mathrm{DNVD}^{299}$-region in combination with the WDD, the exact mechanism by which T300A might impair WDD function still remains elusive. But the structure of the isolated WDD presented here will likely help to dissect this mechanism in more detail in the future.

\section{Conclusion}

In summary, in this work we experimentally identified the domain boundaries of the predicted WDD of ATG16L1 and present the first data for the structure of the WDD. The structure of the WDD was determined to a resolution of $1.55 \AA$ by X-raycrystallography and provides novel insights into the architecture of ATG16L1 and potential interaction surfaces of its C-terminal WDD (Fig. 5). In the future, the biochemical and high-resolution structural data presented here will facilitate a structureassisted mutagenesis approach and the structure determination of WDD in complex with its individual interaction partners to further analyze and dissect the interactions of the WDD with the individual interaction partners in more detail at the cellular, molecular and atomic level.

\section{Materials and Methods}

The Supporting Information contains a detailed description of the protein expression, purification, data processing and structure determination.

\section{Cloning, protein expression and purification}

In short, human ATG16L1 (Uniprot ID-Q676U5) expression constructs were amplified by PCR from a cDNA template (IHS1382-8397581/LIFESEQ2507216, OpenBiosystems) and cloned into a modified pFastBacDual vector with an $\mathrm{N}$-terminal 6xHis tag followed by a TEV protease cleavage site. Mutants were generated by standard mutagenesis PCR. Protein was expressed in BTI-Tn-5B1-4 (HighFive, Invitrogen) insect cells in EX-CELL405 media (Sigma-Aldrich) using the Baculovirus expression system (Invitrogen) and purified via Ni-NTA affinity chromatography and size-exclusion chromatography on a HiLoad 16/60 Superdex 75 column (GE Healthcare) in $20 \mathrm{mM}$ HEPES pH 7.4, $200 \mathrm{mM} \mathrm{NaCl}, 5 \mathrm{mM}$ DTT. Protein was concentrated to $20 \mathrm{mg} / \mathrm{mL}$, aliquots were frozen in liquid $\mathrm{N}_{2}$ and stored at $-80^{\circ} \mathrm{C}$.

\section{Crystallization, data collection and structure determination}

ATG16L1 $1^{303}$ (E354A/K355A/Y607P) was crystallized at a concentration of $10 \mathrm{mg} / \mathrm{ml}$ in $1.61 \mathrm{M}\left(\mathrm{NH}_{4}\right)_{2} \mathrm{SO}_{4}$, $0.09 \mathrm{M} \mathrm{NaCl}, 0.1 \mathrm{M} \mathrm{MES} \mathrm{pH} 6.3$ at $30^{\circ} \mathrm{C}$ using the sitting drop vapour diffusion method. Crystals were cryoprotected by addition of $12 \%$ 2R-3R butane-diol to the crystallization solution. Data were collected at beamline 14.2 at Helmholtz-Zentrum Berlin, BESSY II synchrotron, at a wavelength of $0.9184 \AA$ and a 
temperature of $100 \mathrm{~K}$. The structure was solved by molecular replacement with the published structure of WDR5 (PDB 2CNX) as poly-Ala search model followed by manual rebuilding.

\section{Supporting Information}

The Supporting Information Material 1 file contains a detailed description of experimental procedures, Supporting Information Figures 1-5 and Supporting Information References. Supporting Information Material 2 contains aligned sequences in FASTA format used for Consurf.

\section{Accession Numbers}

Atomic coordinates and structure factors of human ATG16L1 WD40-domain have been deposited in the Protein Data Bank with the accession code 5NUV.

\section{Conflict of Interest}

All the authors have declared no conflict of interest.

\section{Acknowledgments}

X-ray data were collected at beamline 14.2 of BESSY II (Helmholtz-Zentrum Berlin (HZB), Berlin, Germany). We thank HZB for the allocation of synchrotron radiation beamtime. We thank all the beamline staff for their support. Work in the group of A.S. is partially funded by the Helmholtz Association Young Investigator grant number VH-NG-727.

\section{References}

1. Mizushima N (2007) Autophagy: process and function. Genes Dev 21:2861-2873.

2. Yang Z, Klionsky DJ (2009) An overview of the molecular mechanism of autophagy. Curr Top Microbiol Immunol 335:1-32.

3. Paul P, Munz C (2016) Autophagy and mammalian viruses: Roles in immune response, viral replication, and beyond. Adv Virus Res 95:149-195.

4. Ktistakis NT, Tooze SA (2016) Digesting the expanding mechanisms of autophagy. Trends Cell Biol 26:624-635.

5. Deretic V, Saitoh T, Akira S (2013) Autophagy in infection, inflammation and immunity. Nat Rev Immunol 13:722-737.

6. Sica V, Galluzzi L, Bravo-San Pedro JM, Izzo V, Maiuri MC, Kroemer G (2015) Organelle-specific initiation of autophagy. Mol Cell 59:522-539.

7. Komatsu M, Ichimura Y (2010) Selective autophagy regulates various cellular functions. Genes Cells 15: 923-933.

8. Levine B, Mizushima N, Virgin HW (2011) Autophagy in immunity and inflammation. Nature 469:323-335.

9. Deretic V, Kimura T, Timmins G, Moseley P, Chauhan S, Mandell M (2015) Immunologic manifestations of autophagy. J Clin Invest 125:75-84.

10. Jiang X, Overholtzer M, Thompson CB (2015) Autophagy in cellular metabolism and cancer. J Clin Invest 125:47-54.

11. Frake RA, Ricketts T, Menzies FM, Rubinsztein DC (2015) Autophagy and neurodegeneration. J Clin Invest 125:65-74.

12. White $\mathrm{E}$ (2015) The role for autophagy in cancer. $\mathrm{J}$ Clin Invest 125:42-46.
13. Klionsky DJ (2009) Crohn's disease, autophagy, and the Paneth cell. N Engl J Med 360:1785-1786.

14. Nguyen HT, Lapaquette P, Bringer MA, DarfeuilleMichaud A (2013) Autophagy and Crohn's disease. J Innate Immun 5:434-443.

15. Tsukada M, Ohsumi Y (1993) Isolation and characterization of autophagy-defective mutants of Saccharomyces cerevisiae. FEBS Lett 333:169-174.

16. Thumm M, Egner R, Koch B, Schlumpberger M, Straub M, Veenhuis M, Wolf DH (1994) Isolation of autophagocytosis mutants of Saccharomyces cerevisiae. FEBS Lett 349:275-280.

17. Feng Y, He D, Yao Z, Klionsky DJ (2014) The machinery of macroautophagy. Cell Res 24:24-41.

18. Rubinsztein DC, Shpilka T, Elazar Z (2012) Mechanisms of autophagosome biogenesis. Curr Biol 22:R29-R34.

19. Mizushima N, Yoshimori T, Ohsumi Y (2011) The role of Atg proteins in autophagosome formation. Annu Rev Cell Dev Biol 27:107-132.

20. Ichimura Y, Kirisako T, Takao T, Satomi Y, Shimonishi Y, Ishihara N, Mizushima N, Tanida I, Kominami E, Ohsumi M, Noda T, Ohsumi Y (2000) A ubiquitin-like system mediates protein lipidation. Nature 408:488-492.

21. Fujita N, Itoh $\mathrm{T}$, Omori $\mathrm{H}$, Fukuda M, Noda $\mathrm{T}$, Yoshimori T (2008) The Atg16L complex specifies the site of LC3 lipidation for membrane biogenesis in autophagy. Mol Biol Cell 19:2092-2100.

22. Hanada T, Noda NN, Satomi Y, Ichimura Y, Fujioka Y, Takao T, Inagaki F, Ohsumi Y (2007) The Atg12-Atg5 conjugate has a novel E3-like activity for protein lipidation in autophagy. J Biol Chem 282:37298-37302.

23. Romanov J, Walczak M, Ibiricu I, Schuchner S, Ogris E, Kraft C, Martens S (2012) Mechanism and functions of membrane binding by the Atg5-Atg12/Atg16 complex during autophagosome formation. EMBO J 31:4304-4317.

24. Kaufmann A, Beier V, Franquelim HG, Wollert T (2014) Molecular mechanism of autophagic membranescaffold assembly and disassembly. Cell 156:469-481.

25. Otomo C, Metlagel Z, Takaesu G, Otomo T (2013) Structure of the human ATG12 ATG5 conjugate required for LC3 lipidation in autophagy. Nat Struct Mol Biol 20:59-66.

26. Fujioka Y, Noda NN, Nakatogawa H, Ohsumi Y, Inagaki F (2010) Dimeric coiled-coil structure of Saccharomyces cerevisiae Atg16 and its functional significance in autophagy. J Biol Chem 285:1508-1515.

27. Parkhouse R, Ebong IO, Robinson CV, Monie TP (2013) The N-terminal region of the human autophagy protein ATG16L1 contains a domain that folds into a helical structure consistent with formation of a coiled-coil. PLoS One 8:e76237.

28. Dooley HC, Razi M, Polson HE, Girardin SE, Wilson MI, Tooze SA (2014) WIPI2 links LC3 conjugation with PI3P, autophagosome formation, and pathogen clearance by recruiting Atg12-5-16L1. Mol Cell 55:238-252.

29. Itoh T, Fujita N, Kanno E, Yamamoto A, Yoshimori T, Fukuda M (2008) Golgi-resident small GTPase Rab33B interacts with Atg16L and modulates autophagosome formation. Mol Biol Cell 19:2916-2925.

30. Gammoh N, Florey O, Overholtzer M, Jiang X (2013) Interaction between FIP200 and ATG16L1 distinguishes ULK1 complex-dependent and -independent autophagy. Nat Struct Mol Biol 20:144-149.

31. Mizushima N, Kuma A, Kobayashi Y, Yamamoto A, Matsubae M, Takao T, Natsume T, Ohsumi Y, Yoshimori T (2003) Mouse Apg16L, a novel WD-repeat protein, targets to the autophagic isolation membrane with the Apg12-Apg5 conjugate. J Cell Sci 116:16791688 . 
32. Hampe J, Franke A, Rosenstiel P, Till A, Teuber M, Huse K, Albrecht M, Mayr G, De La Vega FM, Briggs J, Gunther S, Prescott NJ, Onnie CM, Hasler R, Sipos B, Folsch UR, Lengauer T, Platzer M, Mathew CG, Krawczak M, Schreiber S (2007) A genome-wide association scan of nonsynonymous SNPs identifies a susceptibility variant for Crohn disease in ATG16L1. Nat Genet 39:207-211.

33. Lassen KG, Kuballa P, Conway KL, Patel KK, Becker CE, Peloquin JM, Villablanca EJ, Norman JM, Liu TC, Heath RJ, Becker ML, Fagbami L, Horn H, Mercer J, Yilmaz OH, Jaffe JD, Shamji AF, Bhan AK, Carr SA, Daly MJ, Virgin HW, Schreiber SL, Stappenbeck TS, Xavier RJ (2014) Atg16L1 T300A variant decreases selective autophagy resulting in altered cytokine signaling and decreased antibacterial defense. Proc Natl Acad Sci U S A 111:7741-7746.

34. Murthy A, Li Y, Peng I, Reichelt M, Katakam AK, Noubade R, Roose-Girma M, DeVoss J, Diehl L, Graham RR, van Lookeren Campagne M (2014) A Crohn's disease variant in Atg1611 enhances its degradation by caspase 3. Nature 506:456-462.

35. Boada-Romero E, Serramito-Gomez I, Sacristan MP, Boone DL, Xavier RJ, Pimentel-Muinos FX (2016) The T300A Crohn's disease risk polymorphism impairs function of the WD40 domain of ATG16L1. Nat Commun 7:11821.

36. Boada-Romero E, Letek M, Fleischer A, Pallauf K, Ramon-Barros C, Pimentel-Muinos FX (2013) TMEM59 defines a novel ATG16L1-binding motif that promotes local activation of LC3. EMBO J 32:566-582.

37. Ramjeet M, Hussey S, Philpott DJ, Travassos LH (2010) 'Nodophagy': New crossroads in Crohn disease pathogenesis. Gut Microbes 1:307-315.

38. Travassos LH, Carneiro LA, Ramjeet M, Hussey S, Kim YG, Magalhaes JG, Yuan L, Soares F, Chea E, Le Bourhis L, Boneca IG, Allaoui A, Jones NL, Nunez G, Girardin SE, Philpott DJ (2010) Nod1 and Nod2 direct autophagy by recruiting ATG16L1 to the plasma membrane at the site of bacterial entry. Nat Immunol 11:55-62.

39. Fujita N, Morita E, Itoh T, Tanaka A, Nakaoka M, Osada Y, Umemoto T, Saitoh T, Nakatogawa H, Kobayashi S, Haraguchi T, Guan JL, Iwai K, Tokunaga F, Saito K, Ishibashi K, Akira S, Fukuda M, Noda T, Yoshimori T (2013) Recruitment of the autophagic machinery to endosomes during infection is mediated by ubiquitin. J Cell Biol 203:115-128.

40. He YJ, McCall CM, Hu J, Zeng Y, Xiong Y (2006) DDB1 functions as a linker to recruit receptor WD40 proteins to CUL4-ROC1 ubiquitin ligases. Genes Dev 20:2949-2954.

41. Hu J, Li G, Qu L, Li N, Liu W, Xia D, Hongdu B, Lin $\mathrm{X}$, Xu C, Lou Y, He Q, Ma D, Chen Y (2016) TMEM166/EVA1A interacts with ATG16L1 and induces autophagosome formation and cell death. Cell Death Dis 7:e2323.

42. Kimura T, Jain A, Choi SW, Mandell MA, Schroder K, Johansen T, Deretic V (2015) TRIM-mediated precision autophagy targets cytoplasmic regulators of innate immunity. J Cell Biol 210:973-989.

43. de Castro E, Sigrist CJ, Gattiker A, Bulliard V, Langendijk-Genevaux PS, Gasteiger E, Bairoch A, Hulo N (2006) ScanProsite: detection of PROSITE signature matches and ProRule-associated functional and structural residues in proteins. Nucleic Acids Res 34: W362-W365.

44. Finn RD, Coggill P, Eberhardt RY, Eddy SR, Mistry J, Mitchell AL, Potter SC, Punta M, Qureshi M, Sangrador-Vegas A, Salazar GA, Tate J, Bateman A (2016) The Pfam protein families database: towards a more sustainable future. Nucleic Acids Res 44:D279_ D285.

45. Letunic I, Doerks T, Bork P (2015) SMART: recent updates, new developments and status in 2015. Nucleic Acids Res 43:D257-D260.

46. Buchan DW, Minneci F, Nugent TC, Bryson K, Jones DT (2013) Scalable web services for the PSIPRED Protein Analysis Workbench. Nucleic Acids Res 41:W349W357.

47. Goldschmidt L, Cooper DR, Derewenda ZS, Eisenberg D (2007) Toward rational protein crystallization: A Web server for the design of crystallizable protein variants. Protein Sci 16:1569-1576.

48. Neer EJ, Schmidt CJ, Nambudripad R, Smith TF (1994) The ancient regulatory-protein family of WDrepeat proteins. Nature 371:297-300.

49. Xu C, Min J (2011) Structure and function of WD40 domain proteins. Protein Cell 2:202-214.

50. Stirnimann CU, Petsalaki E, Russell RB, Muller CW (2010) WD40 proteins propel cellular networks. Trends Biochem Sci 35:565-574.

51. Ma X, Panjikar S, Koepke J, Loris E, Stockigt J (2006) The structure of Rauvolfia serpentina strictosidine synthase is a novel six-bladed beta-propeller fold in plant proteins. Plant Cell 18:907-920.

52. Smith TF, Gaitatzes C, Saxena K, Neer EJ (1999) The WD repeat: a common architecture for diverse functions. Trends Biochem Sci 24:181-185.

53. Ashkenazy H, Abadi S, Martz E, Chay O, Mayrose I, Pupko T, Ben-Tal N (2016) ConSurf 2016: an improved methodology to estimate and visualize evolutionary conservation in macromolecules. Nucleic Acids Res 44: W344-W350.

54. Schuetz A, Allali-Hassani A, Martin F, Loppnau P, Vedadi M, Bochkarev A, Plotnikov AN, Arrowsmith CH, Min J (2006) Structural basis for molecular recognition and presentation of histone H3 by WDR5. EMBO J 25:4245-4252.

55. Julien O, Wells JA (2017) Caspases and their substrates. Cell Death Differ 24:1380-1389. 